Collection SFN 8 (2007) 159

(C) EDP Sciences, Les Ulis

DOI: $10.1051 / \mathrm{sfn}: 2007012$

\title{
Diffusion quasi-élastique des neutrons et simulations de dynamique moléculaire : des techniques complémentaires pour étudier la diffusion dans les zéolithes
}

\author{
H. Jobic ${ }^{1}$ et D.N. Theodorou ${ }^{2}$ \\ ${ }^{1}$ Institut de Recherches sur la Catalyse, CNRS, 2 avenue Albert Einstein, \\ 69626 Villeurbanne, France \\ ${ }^{2}$ School of Chemical Engineering, National Technical University of Athens, Zografou \\ Campus, 15780 Athens, Greece
}

\begin{abstract}
Résumé. La diffusion quasi -élastique des neutrons (QENS) et les simulations de dynamique moléculaire (MD) ont permis une étude quantitative des mouvements moléculaires de fluides purs et complexes adsorbés dans des zéolithes sur des échelles de distances allant de 0.1 à $100 \mathrm{~nm}$ et des gammes de temps de $10 \mathrm{fs}$ à 100 ns. Après une introduction à la mécanique statistique de la diffusion, la toute récente revue intitulée "Quasi-elastic neutron scattering and molecular dynamics simulations as complementary techniques for studying diffusion in zeolites" [1] décrit les principes et les pratiques des mesures de temps de vol ("timeof-flight" (TOF)) de rétrodiffusion ("backscattering" (BS)) et d'échos de spins de neutrons ("neutron spinecho" (NSE)). Les méthodes d'analyse des données obtenues par ces techniques sont aussi détaillées. Les fondements utilisés pour les simulations de dynamique moléculaire sont aussi donnés, en insistant sur la manière de calculer la quantité observable en diffusion quasi-élastique, ainsi que sur le moyen de détecter la signature de la dynamique locale translationnelle anisotrope reliée à l'inhomogénéité périodique du champ d'interaction des cristaux de zéolithe. Des illustrations de ce qui peut être déduit en combinant des données de diffusion de neutrons et les simulations moléculaires concernent des travaux récents sur des problèmes et des systèmes spécifiques : l'auto-diffusion d'alcanes jusqu'à $\mathrm{C}_{16}$ dans des zéolites MFI, l'auto-diffusion du méthane co-adsorbé avec du $n$-butane dans la silicalite-1, et la diffusion de transport de l'azote $\left(\mathrm{N}_{2}\right)$ et du gaz carbonique $\left(\mathrm{CO}_{2}\right)$ dans cette silicalite- 1 .

Les exemples présentés durant le cours de l'école « Surfaces, interfaces, milieux confinés par diffusion de neutrons » sont tous détaillés dans cette revue.
\end{abstract}

\section{Référence}

[1] Jobic, H. and Theodorou, D. N., Micropor. Mesopor. Mater. (in press). 